\title{
Synthetic studies of mycalolide B, an actin-depolymerizing marine macrolide: construction of the tris-oxazole macrolactone using ring-closing metathesis
}

\author{
Masaki Kita, Hidekazu Watanabe, Tomoya Ishitsuka, Yuzo Mogi, and Hideo Kigoshi* \\ Department of Chemistry, Graduate School of Pure and Applied Sciences, University of Tsukuba, 1-1-1 Tennodai, Tsukuba, Ibaraki \\ 305-8571, Japan.
}

\begin{abstract}
Tris-oxazole macrolactone 2, a key intermediate of mycalolide B (1), which has 13 stereogenic centers, was synthesized through the use of ring-closing metathesis (RCM). The $E / Z$ ratio of the RCM product 2 was reversed by the use of $\mathrm{CH}_{2} \mathrm{Cl}_{2}$ and toluene, whereas a cross metathesis reaction yielded the C1-C35 long-chain compound $\mathbf{1 9}$ in a highly E-selective manner. Thus, the loss of flexibility in aliphatic carbon chains and the steric hinderance of $\beta$ - and $\gamma$-substituents of the C20 olefin in the precursor $\mathbf{1 1}$ may affect the stereoselectivity in RCM reactions. (c) 2010 Elsevier Science. All rights reserved.
\end{abstract}

Mycalolide B (1) is a cytotoxic and antifungal macrolide isolated from the marine sponge Mycale sp. It bears a unique tris-oxazole structure and 13 stereogenic centers (Fig. 1). ${ }^{1}$ This compound also inhibits actomyosin $\mathrm{Mg}^{2+}$-ATPase and shows potent actin-depolymerizing activity by sequestering G-actin and forming a 1:1 complex. ${ }^{2}$ Mycalolides can be divided into two characteristic parts: the $\mathrm{C} 1-\mathrm{C} 24$ macrolactone and the C25-C35 side-chain moieties. Studies of the structure-activity relationship ${ }^{3}$ and photo-affinity labeling experiments ${ }^{4}$ have established that the side-chain part of $\mathbf{1}$ is critically important for its ability to bind to and depolymerize actin. Several tris-oxazole macrolides closely related to mycalolides have been isolated, such as ulapualides, ${ }^{5}$ halichondramides, ${ }^{6}$ jaspisamides, ${ }^{7}$ and kabiramides; ${ }^{8}$ all of which exhibit potent actindepolymerizing properties. These agents may be useful for the development of novel pharmacological tools for analyzing actin-mediated cell functions, such as muscle contraction, cell motility, and cytokinesis. Furthermore, it is noteworthy that aplyronine $\mathrm{A}$, which has an actin-binding side-chain moiety similar to mycalolides, exhibits potent antitumor activity in vivo against P388 leukemia and several cancers. ${ }^{9,10}$ Thus, mycalolides and related actin-targeting natural products have great potential as preclinical candidates for use in cancer chemotherapy.

Due to their extraordinary structures and important biological activities, several synthetic studies on trisoxazole-containing macrolides have been reported. ${ }^{11}$ Recently, total syntheses of mycalolide $\mathrm{A}^{12}$ and ulapualide A ${ }^{13}$ have been accomplished, in which Yamaguchi lactonization, cyclization of the central oxazole ring, or intramolecular Horner-Wadsworth-Emmons olefination were used to construct macrocycles. Subsequent studies have shown that olefin metathesis is a useful method for connecting the C19-C20 double bonds in mycalolide analogs. ${ }^{14}$ Here we describe the synthesis of tris-oxazole macrolactone 2, a key synthetic intermediate of mycalolides, through the use of ring-closing metathesis (RCM). We expected that the convergent assembly of three fragments via Ni/Cr-mediated Nozaki-Hiyama-Kishi coupling ${ }^{15}$ at C6-C7, esterification, and RCM at the C19-C20 olefin could efficiently afford 2.
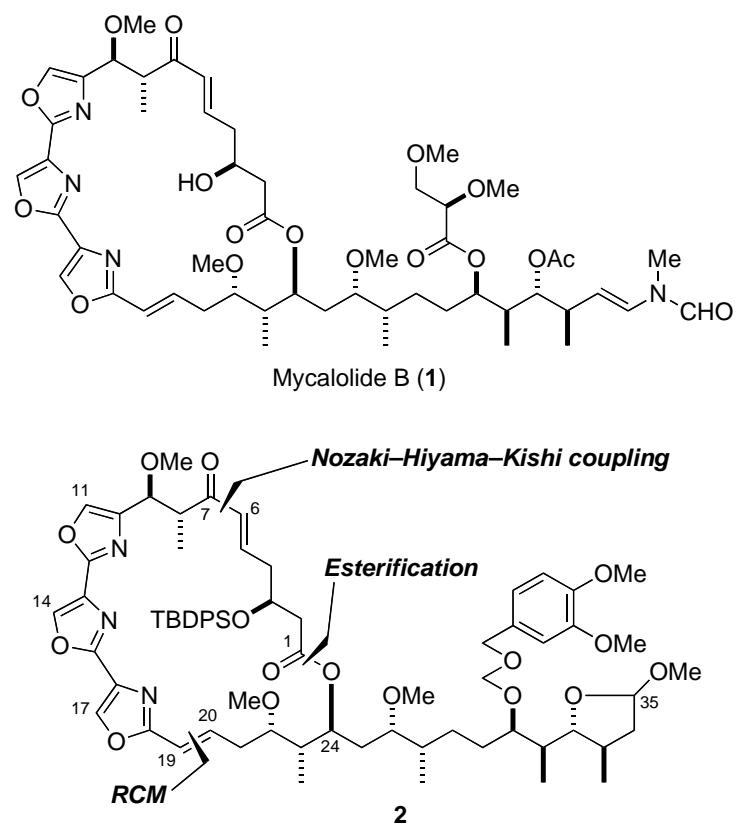

Figure 1. 
The synthesis started with removal of the Boc and acetonide groups of the previously reported oxazole (-)-3 ${ }^{14}$ under acidic conditions, and subsequent condensation with 2-chloroxazole-4-carboxylic acid ${ }^{16}$ afforded amide 4 (77\%, 2 steps) (Scheme 1). Due to the considerable instability of the 2-vinyloxazole moieties under basic and dehydration conditions, we planned to introduce the vinyl group to the oxazole ring after construction of the tris-oxazole structure. Dehydrating cyclization of $\mathbf{4}$ by diethylaminosulfur trifluoride (DAST) ${ }^{17}$ gave an oxazoline intermediate (85\%), which was oxidized with a combination of bromotrichloromethane and 1,8-diazabicycloundec-7-ene (DBU) ${ }^{18}$ at room temperature to give tris-oxazole 5 (98\% based on recovered starting material). ${ }^{19}$ We found that acetonitrile is a better solvent than the conventional $\mathrm{CH}_{2} \mathrm{Cl}_{2}$ in this reaction. Catalytic dihydroxylation of $\mathbf{5}$ with $\mathrm{OsO}_{4}-\mathrm{NMO}$ and Migita-Stille coupling with tri-n-butylvinyltin furnished a vinyloxazole intermediate, and this was transformed into aldehyde 6 via oxidative cleavage of the 1,2-diol with $\mathrm{NaIO}_{4}$ (73\%, 3 steps).

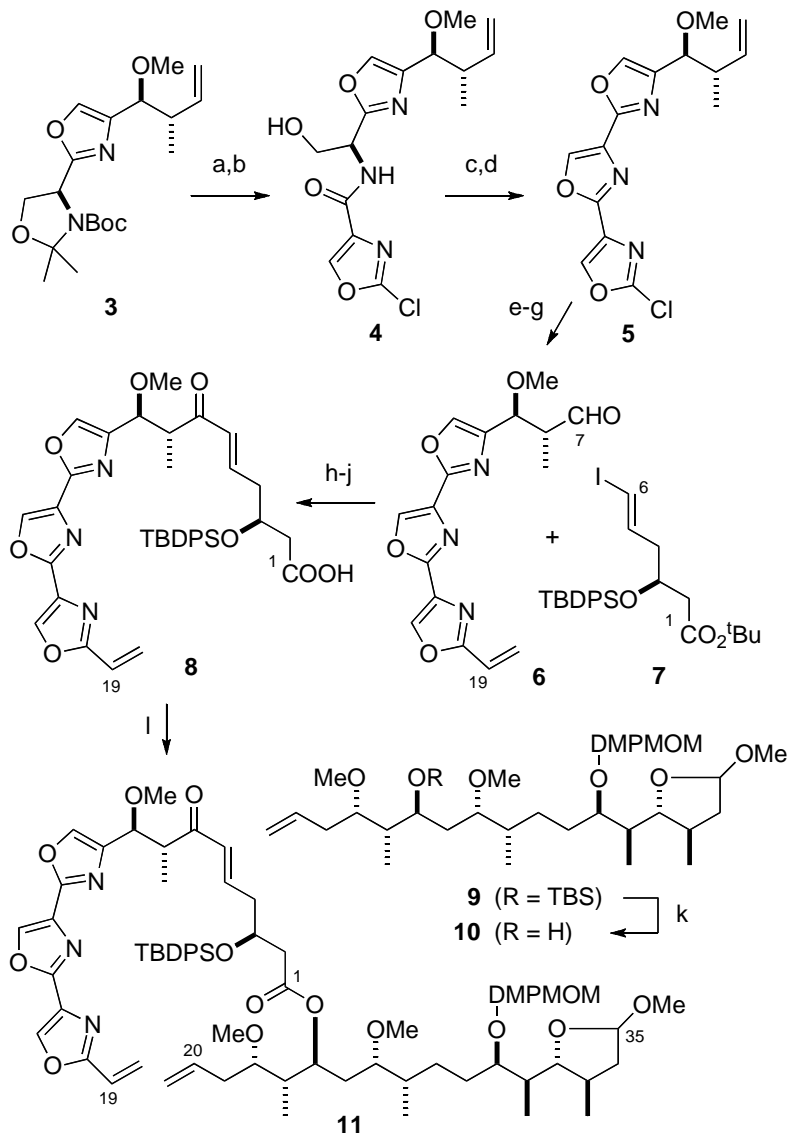

Scheme 1. Synthesis of the RCM precursor 11. Reagents and conditions: (a) $3 \mathrm{M} \mathrm{HCl}$, EtOAc, rt; (b) 2-chlorooxazole-4-carboxylic acid, EDCI·HCl, $\mathrm{HOBt}, \mathrm{Et}_{3} \mathrm{~N}, \mathrm{CH}_{2} \mathrm{Cl}_{2}, 0^{\circ} \mathrm{C}$ to rt, $77 \%$ in 2 steps; (c) DAST, $\mathrm{CH}_{2} \mathrm{Cl}_{2},-78$ to $0{ }^{\circ} \mathrm{C}, 85 \%$; (d) $\mathrm{DBU}, \mathrm{BrCCl}_{3}, \mathrm{MeCN}$, rt, 54\% (98\% brsm); (e) $\mathrm{OsO}_{4}$, NMO, THF- ${ }^{t} \mathrm{BuOH}-\mathrm{H}_{2} \mathrm{O}$, rt; (f) tri-n-butylvinyltin, $\mathrm{PdCl}_{2}\left(\mathrm{PPh}_{3}\right)_{2}$, 1,4-dioxane, reflux; (g) $\mathrm{NaIO}_{4}, \mathrm{EtOH}-\mathrm{H}_{2} \mathrm{O}$, rt, 73\% in 3 steps; (h) 7, $\mathrm{CrCl}_{2}-\mathrm{NiCl}_{2}$, THF-DMF, rt; (i) DMP, pyridine, $\mathrm{CH}_{2} \mathrm{Cl}_{2}, \mathrm{rt}, 71 \%$ in 2 steps; (j) TFA, $\mathrm{CH}_{2} \mathrm{Cl}_{2}, 0{ }^{\circ} \mathrm{C}, 90 \%$; (k) TBAF, THF, $40{ }^{\circ} \mathrm{C}, 97 \%$; (l) 10, MNBA, $\mathrm{Et}_{3} \mathrm{~N}, \mathrm{DMAP}, \mathrm{CH}_{2} \mathrm{Cl}_{2}, \mathrm{rt}, 55 \%$.
Fragment coupling between 6 and vinyl iodide $7^{12}$ by a $\mathrm{Ni} / \mathrm{Cr}$-mediated coupling reaction was followed by oxidation of the C7 allylic alcohol with Dess-Martin periodinane (DMP) ${ }^{20}$ to afford a ketone (71\%, 2 steps), the tert-butyl group of which was removed to give carboxylic acid 8 (90\%). Removal of the tert-butyldimethylsilyl (TBS) group in $\mathbf{9}^{14,3 b, 21}$ by tetra- $n$-butylammonium fluoride (TBAF) gave C20-C35 fragment 10 (97\%), which was condensed with $\mathbf{8}$ by the Shiina procedure ${ }^{22}$ to afford the RCM precursor $\mathbf{1 1}$ in 55\% yield.

With the key intermediate $\mathbf{1 1}$ in hand, RCM reactions were examined (Table 1). First, treatment of 11 with 30 mol\% of 2nd-generation Grubbs catalyst (12) ${ }^{23}$ in degassed refluxing toluene led to decomposition of the starting material and gave a complex mixture (entry 1). We assumed that the low reactivity of $\mathbf{1 1}$ toward RCM reactions would be due to the electron-deficient C19 olefin. To overcome this problem, more thermally-stable and highly-active catalyst was considered. Treatment of $\mathbf{1 1}$ with $30 \mathrm{~mol} \%$ of 2nd-generation Hoveyda-Grubbs catalyst (13) 24 in refluxing $\mathrm{CH}_{2} \mathrm{Cl}_{2}(0.8 \mathrm{mM})$ yielded tris-oxazole lactone 2 as a separable 2:1 mixture of stereoisomers in $30 \%$ yield (entry 2). ${ }^{25-27}$ With the use of toluene as a solvent $(0.9 \mathrm{mM})$, the yield of 2 was improved to $76 \%$, but the $E / Z$-product ratio was changed to $1: 1.2$ (entry 3 ).

Table 1. Ring-closing metathesis of $\mathbf{1 1 .}$

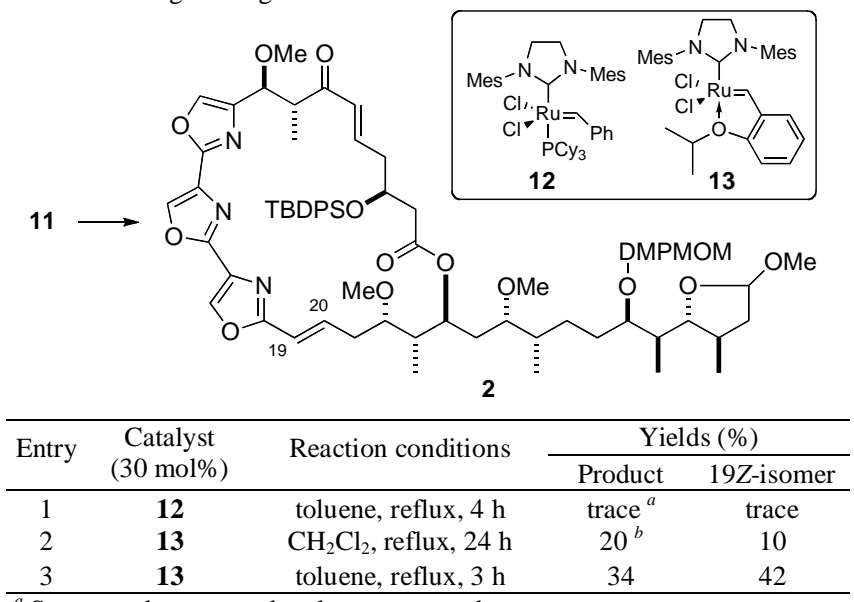

${ }^{a}$ S.m. was decomposed and not recovered.

${ }^{b}$ S.m. was recovered (50\%)

For comparison, we also used a cross-metathesis reaction (Scheme 2). Acidic treatment of cyanide 15 in aqueous $\mathrm{MeOH}$, which was prepared from $(S)$-epichlorohydrin (14), ${ }^{28}$ and protection of the hydroxyl group gave 16 ( $60 \%$ in 2 steps). Ozonolysis of the terminal olefin (80\%) and Takai olefination ${ }^{29}$ gave vinyl iodide $17(66 \%, E / Z=$ 11/1). Nozaki-Hiyama-Kishi coupling between compounds 6 and 17 gave an allylic alcohol (87\%), which was oxidized with DMP to afford the C1-C19 ketone 18 in $84 \%$ yield. In contrast to the RCM reactions, treatment of the C1-C19 segment 18 (1.2 equiv.) and the C20-C35 segment 9 with $50 \mathrm{~mol} \%$ of catalyst 13 in refluxing $\mathrm{CH}_{2} \mathrm{Cl}_{2}$ (7 $\mathrm{mM}$ for 9) for $25 \mathrm{~h}$ yielded the C1-C35 long-chain compound 19 in a highly $E$-selective manner $(66 \%, E / Z=$ $5: 1)^{25,30-32}$ 


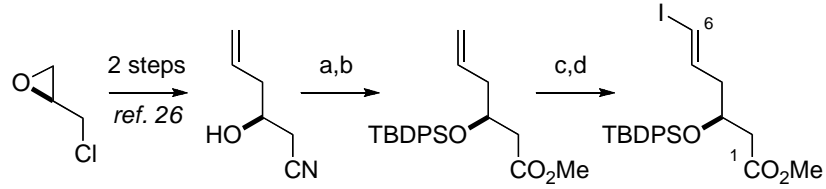

(S)-14 15

16 17

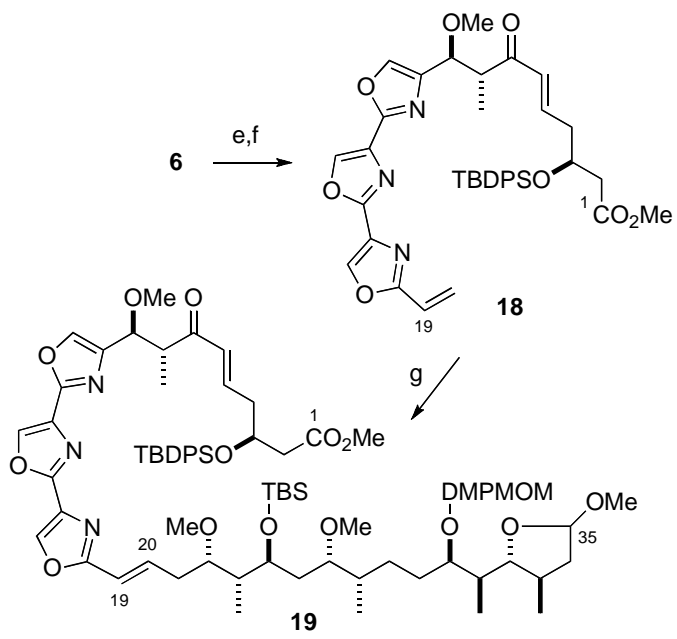

Scheme 2. Cross metathesis reaction. Reagents and conditions: (a) conc. $\mathrm{H}_{2} \mathrm{SO}_{4}, \mathrm{MeOH}-\mathrm{H}_{2} \mathrm{O}$, reflux; (b) TBDPSCl, imidazole, DMF, rt, 60\% in 2 steps; (c) $\mathrm{O}_{3}, \mathrm{CH}_{2} \mathrm{Cl}_{2},-78^{\circ} \mathrm{C}$, then $\mathrm{Me}_{2} \mathrm{~S},-78{ }^{\circ} \mathrm{C}$ to rt, $80 \%$; (d) $\mathrm{CrCl}_{2}$, $\mathrm{CHI}_{3}$, 1,4-dioxane-THF, rt, 65\%; (e) 17, $\mathrm{CrCl}_{2}-\mathrm{NiCl}_{2}, \mathrm{THF}-\mathrm{DMF}$, rt, 87\%; (f) DMP, pyridine, $\mathrm{CH}_{2} \mathrm{Cl}_{2}, \mathrm{rt}, 84 \%$; (g) 9, 13 (50 mol\%), $\mathrm{CH}_{2} \mathrm{Cl}_{2}$, reflux, $55 \%$ with $11 \%$ of $19 Z$-isomer.

Our work demonstrated that the RCM reaction of $\mathbf{1 1}$ proceeded with low stereoselectivity, unlike the cross-metathesis reaction of 18 . The $E / Z$ ratios did not significantly change during the course of the metathesis reactions, and thus the formation of $\mathrm{C}=\mathrm{C}$ bonds in $\mathbf{2}$ and $\mathbf{1 9}$ would take place under kinetic control. In the ruthenocyclobutane intermediate for the desired 19E-isomer of 2, the oxazole rings and C21-C35 alkyl chain are located in an anti-orientation. Due to the rigidness of the tris-oxazole and $\alpha, \beta$-unsaturated ketone moieties, the anti-ruthenocyclobutane intermediate would be more strained than the syn-intermediate, which may affect the stereoselectivity in RCM reactions.

In conclusion, we achieved the synthesis of tris-oxazole macrolactone $\mathbf{2}$ through the use of RCM reactions as a key step, which includes all of the 13 stereogenic centers and the whole carbon framework of mycalolide B (1). Also, this key intermediate possesses a common framework for mycalolides and related actin-depolymerizing tris-oxazole macrolides. Studies on the total synthesis of mycalolide B (1) as well as on the stereoselectivity of RCM reactions, and especially solvent effects, are currently underway.

\section{Acknowledgements}

Support was provided by JSPS via Grants-in-Aids for Scientific Research (21681028 and 21651091 for M.K., and
20310129 for H.K.), by the Kato Memorial Bioscience Foundation, and by the Uehara Memorial Foundation.

\section{References and Notes}

1 (a) Fusetani, N.; Yasumuro, K.; Matsunaga, S.; Hashimoto, K. Tetrahedron Lett. 1989, 30, 2809; (b) Matsunaga, S.; Liu, P.; Celatka, C. A.; Panek, J. S.; Fusetani, N. J. Am. Chem. Soc. 1999, 121, 5605.

2 (a) Hori, M.; Saito, S.; Shin, Y.; Ozaki, H.; Fusetani, N.; Karaki, H. FEBS Lett. 1993, 322, 151; (b) Saito, S.; Watabe, S.; Ozaki, H.; Fusetani, N.; Karaki, H. J. Biol. Chem. 1994, 269, 29710

3 (a) Suenaga, K.; Miya, S.; Kuroda, T.; Handa, T.; Kanematsu, K.; Sakakura, A.; Kigoshi, H. Tetrahedron Lett. 2004, 45, 5383; (b) Suenaga, K.; Kimura, T.; Kuroda, T.; Matsui, K.; Miya, S.; Kuribayashi, S.; Sakakura, A.; Kigoshi, H. Tetrahedron 2006, 62, 8278.

4 Kuroda, T.; Suenaga, K.; Sakakura, A.; Handa, T.; Okamoto, K.; Kigoshi, H. Bioconjugate Chem. 2006, 17, 524.

5 Roesener, J. A.; Scheuer, P. J. J. Am. Chem. Soc. 1986, 108, 846.

6 (a) Matsunaga, S.; Fusetani, N.; Hashimoto, K.; Koseki, K.; Noma, M.; Noguchi, H.; Sankawa, U. J. Org. Chem. 1989, 54, 1360; (b) Kernan, M. R.; Molinski, T. F.; Faulkner, D. J. J. Org. Chem. 1988, 53, 5014.

7 Kobayashi, J.; Murata, O.; Shigemori, H. J. Nat. Prod. 1993, 56, 787.

8 (a) Matsunaga, S.; Fusetani, N.; Hashimoto, K. J. Am. Chem. Soc. 1986, 108, 847; (b) Matsunaga, S.; Fusetani, N.; Hashimoto, K.; Koseki, K.; Noma, M.; Noguchi, H.; Sankawa, U. J. Org. Chem. 1989, 54, 1360; (c) Tanaka, J.; Yan, Y.; Choi, J.; Bai, J.; Klenchin, V. A.; Rayment, I.; Marriott, G. Proc. Natl. Acad. Sci. USA 2003, 100, 13851; (d) Klenchin, V. A.; Allingham, J. S.; King, R.; Tanaka, J.; Marriott, G.; Rayment, I. Nat. Struct. Biol. 2003, 10, 1058.

9 (a) Yamada, K.; Ojika, M.; Ishigaki, T.; Yoshida, Y.; Ekimoto, H.; Arakawa, M. J. Am. Chem. Soc. 1993, 115, 11020; (b) Ojika, M.; Kigoshi, H.; Ishigaki, T.; Tsuboi, T.; Ogawa, T.; Yamada, K. J. Am. Chem. Soc. 1994, 116, 7441; (c) Kigoshi, H.; Suenaga, K.; Mutou, T.; Ishigaki, T.; Atsumi, T.; Ishiwata, H.; Sakakura, A.; Ogawa, T.; Ojika, M.; Yamada, K. J. Org. Chem. 1996, 61, 5326; (d) Hirata, K.; Muraoka, S.; Suenaga, K.; Kuroda, T.; Kato, K.; Tanaka, H.; Yamamoto, M.; Takata, M.; Yamada, K.; Kigoshi, H. J. Mol. Biol. 2006, 356, 945.

10 Reviews: (a) Ojika, M.; Kigoshi, H.; Yoshida, Y.; Ishigaki, T.; Nisiwaki, M.; Tsukada, I.; Arakawa, M.; Ekimoto, H.; Yamada, K. Tetrahedron 2007, 63, 3138; (b) Yamada, K.; Ojika, M.; Kigoshi, H.; Suenaga, K. Nat. Prod. Rep. 2009, 26, 27.

11 Reviews: (a) Yeung, K. -S.; Paterson, I. Angew. Chem. Int. Ed. 2002, 41, 4632; (b) Chattopadhyay, S. K.; Pattenden, G. J. Chem. Soc., Perkin Trans 1, 2000, 2429, and references are therein.

12 (a) Liu, P.; Panek, J. S. J. Am. Chem. Soc. 2000, 122, 1235; (b) Panek, J. S.; Liu, P. J. Am. Chem. Soc. 2000, 122, 11090.

13 (a) Pattenden, G.; Ashweek, N. J.; Baker-Glenn, C. A. G.; Walker, G. M.; Yee, J. G. K. Angew. Chem. Int. Ed. 2007, 46, 4359. (b) Chattopadhyay, S. K.; Pattenden, G. Tetrahedron Lett. 1998, 39, 6095.

14 Kimura, T.; Kuribayashi, S.; Sengoku, T.; Matsui, K.; Ueda, S.; Hayakawa, I.; Suenaga, K.; Kigoshi, H. Chem. Lett. 2007, 36, 1490.

15 (a) Jin, H.; Uenishi, J.; Christ, W. J.; Kishi, Y. J. Am. Chem. Soc. 1986, 108, 5644; (b) Takai, K.; Tagashira, M.; Kuroda, 
T.; Oshima, K.; Utimoto, K.; Nozaki, H. J. Am. Chem. Soc. 1986, 108, 6048; (c) Okude, Y.; Hirano, S.; Hiyama, T.; Nozaki, H. J. Am. Chem. Soc. 1977, 99, 3179.

16 (a) Young, G. L.; Smith, S. A.; Taylor, R. J. K. Tetrahedron Lett. 2004, 45, 3797; (b) Grank, G.; Fouris, M. J. J. Med. Chem. 1971, 14, 1075.

17 Phillips, A. J.; Uto, Y.; Wipf, P.; Reno, M. J.; Williams, D. R. Org. Lett. 2000, 2, 1165.

18 Williams, D. R.; Lowder, P. D.; Gu, Y. -G.; Brooks, D. A. Tetrahedron Lett. 1997, 38, 331.

$19 \mathrm{NiO}_{2}$ oxidation of oxazoline intermediate also afforded $\mathbf{8}$, but low yield $(\sim 30 \%)$ and significant loss of starting material recovery, probably due to the strong coordination of bis- or tris-oxazole nitrogen atoms to nickel atom.

20 Dess, D. B.; Martin, J. C. J. Am. Chem. Soc. 1991, 113, 7277.

21 Although configuration of the C35 acetal carbon in $\mathbf{9}$ has not been determined, $\mathbf{9}$ is a single stereoisomer. See ref. $3 \mathrm{~b}$.

22 (a) Shiina, I.; Kubota, M.; Ibuka, R. Tertahedron Lett. 2002, 43, 7535; (b) Shiina, I.; Kubota, M.; Oshiumi, H.; Hashizume, M. J. Org. Chem. 2004, 69, 1822.

23 (a) Scholl, M.; Ding, S.; Lee, C. W.; Grubbs, R. H. Org. Lett. 1999, 1, 953; (b) Chatterjee, A. K.; Choi, T. -L.; Sanders, D. P.; Grubbs, R. H. J. Am. Chem. Soc. 2003, 125, 11360.

24 (a) Kingsbury, J. S.; Harrity, J. P. A.; Bonitatebus, P. J.; Hoveyda, A. H. J. Am. Chem. Soc. 1999, 121, 791; (b) Garber, S. B.; Kingsbury, J. S.; Gray, B. L.; Hoveyda, A. H. J. Am. Chem. Soc. 2000, 122, 8168.

25 The stereochemistry of the C19 olefins in $\mathbf{2}$ and $\mathbf{1 9}$ was established based on ${ }^{3} J_{\mathrm{H} 19, \mathrm{H} 20}$ values $(15.8$ and $15.9 \mathrm{~Hz})$. In contrast, the ${ }^{3} J_{\mathrm{H} 19, \mathrm{H} 20}$ values of $19 \mathrm{Z}-2$ and $19 \mathrm{Z}-19$ were 11.4 and $11.3 \mathrm{~Hz}$, respectively.

26 Spectral data for 2: $R_{f} 0.12$ (hexane/EtOAc $=1 / 1$ ); $[\alpha]_{\mathrm{D}}{ }^{24}$ -26.2 (c 0.030, $\mathrm{CHCl}_{3}$ ); ${ }^{1} \mathrm{H}$ NMR $\left(500 \mathrm{MHz}, \mathrm{CDCl}_{3}\right) \delta 8.11$ (s, 1H, H-14), 8.06 (s 1H, H-17), 7.71-7.67 (m, 4H, $\left.-\mathrm{Si}\left({ }^{t} \mathrm{Bu}\right) P h_{2}\right), \quad 7.66 \quad(\mathrm{~s}, 1 \mathrm{H}, \mathrm{H}-11), \quad 7.42-7.34 \quad(\mathrm{~m}, \quad 6 \mathrm{H}$, $\left.-\mathrm{Si}\left({ }^{t} \mathrm{Bu}\right) P h_{2}\right), 7.15-7.06$ (m, 2H, H-5, H-20), 6.90-6.80 (m, $\left.3 \mathrm{H},-\mathrm{C}_{6} \mathrm{H}_{3}(\mathrm{OMe})_{2}\right), 6.32(\mathrm{~d}, J=15.8 \mathrm{~Hz}, 1 \mathrm{H}, \mathrm{H}-19), 5.90$ (d, $J=16.2 \mathrm{~Hz}, 1 \mathrm{H}, \mathrm{H}-6), 5.12(\mathrm{~m}, 1 \mathrm{H}, \mathrm{H}-24), 4.86$ (d, $J=4.7$ $\mathrm{Hz}, 1 \mathrm{H}, \mathrm{H}-35$ ), 4.81-4.79 (AB quart, $J=11.2 \mathrm{~Hz}, 2 \mathrm{H}$, $\left.-\mathrm{OCH}_{2} \mathrm{O}-\right)$, 4.56 (s, $\left.2 \mathrm{H},-\mathrm{OCH}_{2} \mathrm{Ar}\right), 4.43(\mathrm{~m}, 1 \mathrm{H}, \mathrm{H}-22), 4.37$ $(\mathrm{d}, J=9.5 \mathrm{~Hz}, 1 \mathrm{H}, \mathrm{H}-9), 4.28(\mathrm{~m}, 1 \mathrm{H}, \mathrm{H}-3), 4.19(\mathrm{~m}, 1 \mathrm{H}$, H-26), 4.02 (m, 1H, H-30), 3.87 (s, 3H, $-\mathrm{OMe}$ ), 3.86 (s, 3H, $-\mathrm{OMe}$ ), 3.54 (m, 1H, H-32), 3.26 (s, 3H, $-\mathrm{OMe}), 3.24$ (s, 3H, $-\mathrm{OMe}$ ), 3.22 (s, 3H, $-\mathrm{OMe}), 3.10(\mathrm{~s}, 3 \mathrm{H},-\mathrm{OMe}), 2.98(\mathrm{~m}$, $1 \mathrm{H}), 2.74-2.70(\mathrm{~m}, 2 \mathrm{H}), 2.45-2.28(\mathrm{~m}, 2 \mathrm{H}), 1.80(\mathrm{~m}, 4 \mathrm{H})$, $1.66-1.40(\mathrm{~m}, 10 \mathrm{H}), 1.08(\mathrm{~d}, J=6.6 \mathrm{~Hz}, 3 \mathrm{H}), 1.03(\mathrm{~s}, 9 \mathrm{H}$, $\left.-\mathrm{Si}\left({ }^{\mathrm{t}} \mathrm{Bu}\right) \mathrm{Ph}_{2}\right), 0.88-0.77$ (m, 12H); IR $\left(\mathrm{CHCl}_{3}\right)$ 2930, 1733,
1654, 1516, 1458, 1381, 1262, 1106, 1027, 755, $704 \mathrm{~cm}^{-1}$; HRMS (ESI) $\mathrm{m} / \mathrm{z} 1282.6232$ (calcd for $\mathrm{C}_{70} \mathrm{H}_{93} \mathrm{~N}_{3} \mathrm{NaO}_{18} \mathrm{Si}$ $\left.[\mathrm{M}+\mathrm{Na}]^{+}, \Delta+1.0 \mathrm{mmu}\right)$.

27 The dimer of $\mathbf{1 1}$ was not formed.

28 Hanazawa, T.; Okamoto, T.; Sato, F. Tetrahedron Lett. 2001, 42, 5455.

29 (a) Takai, K.; Nitta, K.; Utimoto, K. J. Am. Chem. Soc. 1986, 108, 7408; (b) Takai, K.; Kimura, K.; Kuroda, T.; Hiyama, T.; Nozaki, H. Tetrahedron Lett. 1983, 24, 5281.

30 Spectral data for 19: $R_{f} 0.10$ (hexane/EtOAc $=1 / 1$ ); $[\alpha]_{\mathrm{D}}{ }^{25}$ -15.5 (c 0.415, $\left.\mathrm{CHCl}_{3}\right) ;{ }^{1} \mathrm{H}$ NMR $\left(270 \mathrm{MHz}, \mathrm{CDCl}_{3}\right) \delta 8.33$ (s, 1H, H-14), 8.28 (s, 1H, H-17), 7.69 (s, 1H, H-11), 7.69-7.64 (m, 4H, $\left.-\mathrm{Si}\left({ }^{\mathrm{t}} \mathrm{Bu}\right) \mathrm{Ph}_{2}\right), \quad 7.42-7.36 \quad(\mathrm{~m}, \quad 6 \mathrm{H}$, $\left.-\mathrm{Si}\left({ }^{t} \mathrm{Bu}\right) \mathrm{Ph}_{2}\right), 6.93-6.75$ (m, 5H, H-5, H-20, $-\mathrm{C}_{6} \mathrm{H}_{3}(\mathrm{OMe})_{2}$ ), 6.44 (d, $J=15.9 \mathrm{~Hz}, 1 \mathrm{H}, \mathrm{H}-19), 6.11$ (d, $J=15.7 \mathrm{~Hz}, 1 \mathrm{H}$, $\mathrm{H}-6), 4.89$ (d, $J=4.9 \mathrm{~Hz}, 1 \mathrm{H}, \mathrm{H}-35), 4.83\left(\mathrm{~s}, 2 \mathrm{H},-\mathrm{OCH}_{2} \mathrm{O}-\right.$ ), 4.59 (s, 2H, $\left.-\mathrm{OCH}_{2} \mathrm{Ar}\right), 4.39$ (d, $\left.J=10.0 \mathrm{~Hz}, 1 \mathrm{H}, \mathrm{H}-9\right), 4.32$ (m, 1H, H-3), 4.07 (m, 1H, H-30), 3.92 (m, 1H, H-24), 3.89 (s, $3 \mathrm{H},-\mathrm{OMe}$ ), 3.87 (s, 3H, $-\mathrm{OMe}$ ), 3.58 (dd, $J=6.8,9.5 \mathrm{~Hz}, 1 \mathrm{H}$, $\mathrm{H}-22$ ), 3.56 (s, 3H, $-\mathrm{OMe}$ ), 3.44 (m, 1H, H-32), 3.36 (s, 3H, $-\mathrm{OMe}$ ), 3.33 (s, 3H, $-\mathrm{OMe}$ ), 3.29 (s, 3H, $-\mathrm{OMe}$ ), $3.20(\mathrm{~m}, 1 \mathrm{H}$, $\mathrm{H}-26), 3.17$ (s, 3H, -OMe), $3.03(\mathrm{~m}, 1 \mathrm{H}), 2.60(\mathrm{~m}, 1 \mathrm{H})$, 2.53-2.39 (m, 5H), 2.30-2.05 (m, 3H), 1.87-1.73 (m, 2H), 1.69-1.12 (m, 7H), 1.11 (d, $J=6.5 \mathrm{~Hz}, 3 \mathrm{H}), 1.04$ (s, 9H, $\left.-\mathrm{Si}\left({ }^{\mathrm{t}} \mathrm{Bu}\right) \mathrm{Ph}_{2}\right), 0.95$ (d, $\left.J=6.8 \mathrm{~Hz}, 3 \mathrm{H}\right), 0.89$ (d, $J=7.6 \mathrm{~Hz}$, $3 \mathrm{H}), 0.87-0.82$ (m, 6H), 0.84 (s, 9H, $\left.-\mathrm{Si}\left({ }^{t} \mathrm{Bu}\right) \mathrm{Me}_{2}\right), 0.00$ (s, $\left.3 \mathrm{H},-\mathrm{Si}\left({ }^{t} \mathrm{Bu}\right) M e_{2}\right),-0.06$ (s, 3H, $\left.-\mathrm{Si}\left({ }^{t} \mathrm{Bu}\right) M e_{2}\right) ;{ }^{13} \mathrm{C}$ NMR $(150$ $\left.\mathrm{MHz}, \mathrm{CDCl}_{3}\right) \delta 201.8,171.4,161.9,156.1,155.5,148.9$, 148.5, 142.7, 139.8, 138.6 (2C), 138.5, 137.2, 135.9 (2C), 135.8 (2C), 133.4 (2C), 132.9, 131.5, 130.7, 130.5, 129.9, $129.8,127.7$ (2C), 127.6 (2C), 120.5, 118.0, 111.3, 110.8, 104.6, 94.4, 87.1, 82.5, 82.1, 78.4, 77.5, 69.6, 69.4, 69.2, 57.6, 57.2, 56.9, 55.9, 55.8, 54.5, 51.5, 46.9, 43.4, 43.1, 42.5, 41.5, 40.2, 35.9, 34.6, 33.7, 32.6, 30.6, 26.9, 26.9, 26.9, 26.8, 25.8, 25.8, 25.8, 20.1, 19.2, 18.0, 15.8, 14.1, 9.2, 8.8, -4.1, -4.7; IR $\left(\mathrm{CHCl}_{3}\right)$ 1733, 1664, 1517, 1464, 1380, 1260, 1096, 1029, 919, $823 \mathrm{~cm}^{-1}$; HRMS (ESI) $\mathrm{m} / \mathrm{z} 725.8638$ (calcd for $\left.\left(\mathrm{C}_{77} \mathrm{H}_{111} \mathrm{~N}_{3} \mathrm{Na}_{2} \mathrm{O}_{17} \mathrm{Si}_{2}\right) / 2[\mathrm{M}+2 \mathrm{Na}]^{2+}, \Delta+1.4 \mathrm{mmu}\right)$.

31 The C20-C35 dimer was afforded in 15\% yield, and the dimer of $\mathbf{1 8}$ was not formed.

32 Model reactions for the cross-metathesis of 2-vinyloxazole derivatives using catalyst $\mathbf{1 3}$ in toluene at $40{ }^{\circ} \mathrm{C}$ also preferentially yielded $E$-isomer, but the selectivity was lower than in the case of $\mathrm{CH}_{2} \mathrm{Cl}_{2}(E / Z=2.0 \sim 1.5: 1)$. Thus, the difference of solvent $\left(\mathrm{CH}_{2} \mathrm{Cl}_{2}\right.$ and toluene) rather than reaction temperature may affect the stereoselectivity in the RCM reactions of $\mathbf{1 1 .}$
Keywords: Actin-depolymerizing compound; Ring-closing metathesis; Tris-oxazole macrolide; Synthesis of marine natural products.

* Corresponding author. Tel./fax: +81 298534313.

E-mail address: kigoshi@chem.tsukuba.ac.jp (H. Kigoshi). 